\title{
ANALYSIS OF OXIDISED HEAVY PARAFFININC PRODUCTS BY HIGH TEMPERATURE COMPREHENSIVE TWO-DIMENSIONAL GAS CHROMATOGRAPHY
}

\author{
H. Potgieter ${ }^{1, *}$, R. Bekker ${ }^{1}$, J Beigley ${ }^{1}$, E. Rohwer ${ }^{2}$ \\ 1. Operations and Analytics Department, Group Technology R\&T, Sasolburg, 1947, South Africa. \\ 2. Department of Chemistry, University of Pretoria, Hatfield, Pretoria, 0002, South Africa
}

*Corresponding author: johann.potgieter@sasol.com,

tel: +27169602478, cell: +27822004373, fax: +27112193768

\section{HIGHLIGHTS}

- The separation of oxygenates in oxidised heavy paraffinic fractions by HT-GC $\times \mathrm{GC}$ is reported for the first time.

- HT-GC $\times$ GC column combinations for the separation of oxygenates in oxidised products were optimised.

- The advantages of HT-GC $\times$ GC in monitoring oxidation reactions of heavy paraffinic fraction samples is Illustrated.

- Selective relative quantification of oxygenate classes and obtaining the concentrations by carbon number.

- Advantage of replacing separate titrimetric procedures by a single HT-GC $\times$ GC analysis is clearly Illustrated.

\begin{abstract}
Heavy petroleum fractions are produced during crude and synthetic crude oil refining processes and they need to be upgraded to useable products to increase their market value. Usually these fractions are upgraded to fuel products by hydrocracking, hydroisomerization and hydrogenation processes. These fractions are also upgraded to other high value commercial products like lubricant oils and waxes by distillation, hydrogenation, and oxidation and/or blending. Oxidation of hydrogenated heavy paraffinic
\end{abstract}


fractions produces high value products that contain a variety of oxygenates and the characterization of these heavy oxygenates is very important for the control of oxidation processes. Traditionally titrimetric procedures are used to monitor oxygenate formation, however, these titrimetric procedures are tedious and lack selectivity toward specific oxygenate classes in complex matrices. Comprehensive two-dimensional gas chromatography $(\mathrm{GC} \times \mathrm{GC})$ is a way of increasing peak capacity for the comprehensive analysis of complex samples. Other groups have used HT-GC×GC to extend the carbon number range attainable by $\mathrm{GC} \times \mathrm{GC}$ and have optimised $\mathrm{HT}-\mathrm{GC} \times \mathrm{GC}$ parameters for the separation of aromatics, nitrogen-containing compounds as well as sulphur-containing compounds in heavy petroleum fractions. HT-GC×GC column combinations for the separation of oxygenates in oxidised heavy paraffinic fractions are optimised in this study. The advantages of the HT-GC×GC method in the monitoring of the oxidation reactions of heavy paraffinic fraction samples are illustrated.

\section{Keywords:}

High Temperature Comprehensive two-dimensional gas chromatography; oxygencontaining compounds, oxidised heavy paraffinic fractions.

\section{INTRODUCTION}

Heavy petroleum fractions are produced during crude and synthetic crude oil refining processes. To increase the value of these heavy petroleum fractions, they need upgrading to various marketable products. Usually these fractions are upgraded into fuel products to address the increasing fuel demand using hydrocracking, hydroisomerization and hydrogenation processes [1, 2]. Hydrocracking processes are used to reduce the boiling range of these fractions whilst hydroisomerization processes are used to improve the cold flow properties of fuel. Hydrotreatment processes are used to improve the quality of upgraded products. These heavy petroleum fractions are not only used for the production of fuel but other high value commercial products like lubricant oils and waxes can also be produced by distillation, hydrogenation, and oxidation and/or blending [3]. Oxidation of hydrogenated heavy paraffinic fractions produces high value products that contain a variety of oxygenates. The characterization and monitoring of these heavy oxygenates is very important for the control of oxidation processes. Traditionally titrimetric procedures [4 - 6] are used to determine the acid, alcohol and ester concentrations in an attempt to 
monitor oxygenate formation and the use of these methods for the control of oxidation processes are reported in previous studies [7 - 9]. These titrimetric procedures are tedious and lack selectivity toward specific oxygenate classes in complex matrices. It is stated in the standard test method for acid number [4] that the compounds considered to have acidic characteristics include organic acids, esters, phenolic compounds and lactones whilst the standard test method for hydroxyl groups [6] is not suitable for determination of hydroxyl groups attached to tertiary carbon atoms. Another shortcoming of these procedures is that only total concentrations for these oxygenate classes are determined and values are not reported per carbon number. The selective monitoring of oxygenates during the oxidation of heavy paraffinic fractions will be a great advantage in the control of these processes.

Gas chromatography with an oxygen selective detector (GC-O-FID) has been used by other groups to determine the oxygenates in a complex hydrocarbon matrix [10] and the use of electrospray ionization Fourier transform ion cyclotron resonance mass spectrometry (ESI FT-ICR MS) and gas chromatography mass spectrometry (GC-MS) for the identification of oxygen compounds in shale oil and coal tar was reported by Geng et al. [11]. The applicability of these techniques to oxidised heavy paraffinic fractions is yet to be investigated and the characterization of these samples remains a difficult analytical challenge. Firstly, as a rule only a limited range of relatively small and non-polar molecules can be analysed directly by gas chromatography (GC) and secondly, the number of hydrocarbon isomers increase with increasing carbon number [12 - 13]. Many attempts have been made to address the first challenge of extending the range of heavy molecules that can be analysed by GC. One way to address the first challenge involves the transformation of the heavy molecules present in these fractions to species that can be analysed directly by GC by the addition of a pre-treatment step. These include derivatization reactions [14] that involve the transformation of non-volatile heteroatomic molecules into elutable species and pyrolysis [15] that involves the destruction of the samples into smaller fragment-molecules prior to GC analysis. Another important method regularly employed to extend the range of heavy molecules analysable by GC is hightemperature gas chromatography (HT-GC). In HT-GC, heavy molecules are not transformed prior to GC analysis but their elution is improved by the extension of the conventional separation temperature and the adaptation of chromatographic conditions [12]. The difference between GC and HT-GC is not well defined; however, various authors have stated that GC separations with final temperatures higher than $340^{\circ} \mathrm{C}[12$ - 13] can 
be defined as HT-GC. HT-GC is already an established technique and allows for the analysis of a wide molecular weight range ( 100 to >1400 Da, equivalent to alkanes from C7 to >C100) [16 - 17]. The use of HT-GC instrumentation is critical for analysis at temperatures higher than $340^{\circ} \mathrm{C}$ and column and stationary phase technologies need careful consideration. HT-GC analysis requires metal columns or fused silica columns with a special protective coating to withstand these high temperatures. Stationary phase endcapping and the quality of the surface chemistry of the underlying fused silica are also very important aspects to reduce column bleeding in HT-GC [18 - 19]. Due to the lower temperature limits of polar polymer stationary phases compared to non-polar stationary phases, HT-GC is mostly limited to non-polar stationary phases. The use of cool oncolumn or programmed temperature vaporizing (PTV) injectors in HT-GC is also critical to eliminate discrimination towards high-boiling compounds observed during the injection step when split/splitless injectors are used [16 - 17].

The extension of the molecular range that can be analysed by HT-GC is not only reliant on the higher temperatures achievable but also involves the adaptation of chromatographic conditions. These adaptive conditions include the use of short columns with a reduced film thickness, the latter to achieve lower elution temperatures due to the high gas phase ratio. Despite HT-GC being an established technique, chromatographic resolution is often compromised and peak overlap is a major concern when complex samples are analysed. Comprehensive two-dimensional gas chromatography is a way of increasing peak capacity and $\mathrm{GC} \times \mathrm{GC}$ is a powerful tool to analyse complex petrochemical samples. In $\mathrm{GC} \times \mathrm{GC}$ the peak capacity is increased by combining two chromatographic columns with different separation mechanisms [20] and during a single $\mathrm{GC} \times \mathrm{GC}$ analysis the entire sample is subjected to two independent GC separations. The resulting $\mathrm{GC} \times \mathrm{GC}$ chromatogram provides a great deal of information on molecular composition of these samples and also on the presence of different hydrocarbon classes [21]. Until quite recently, the use of $\mathrm{GC} \times \mathrm{GC}$ was limited to the analysis of the molecular range attainable by normal GC analysis. The extension of $\mathrm{GC} \times \mathrm{GC}$ into the HT-GC realm would be of great value as it would reduce the extent of peak overlap observed when analysing oxidised heavy paraffinic fractions. Unfortunately, the extension of $\mathrm{GC} \times \mathrm{GC}$ to $\mathrm{HT}-\mathrm{GC}$ temperatures is limited by the thermal stability of polar columns. Additionally, heavy compounds are often not re-volatilized effectively during the modulation step. These limitations were addressed by other research groups [21 - 22] showing that flow programming in a cryogenic nitrogen modulator improved the desorption of heavy compounds whilst Dutriez et al. [12] reported 
on the evaluation of various column sets utilising mid polarity columns with higher temperature stabilities to do $\mathrm{GC} \times \mathrm{GC}$ at HT-GC temperatures. Dutriez selected an experimental set of capillary columns based on stationary phase chemistry, column diameter, film thickness and column lengths. The results showed that with the correctly adapted parameters, compounds can elute earlier from the first dimension column to reach the second dimension column at lower temperatures. It was concluded that column dimensions are one of the most critical parameters to optimize in HT-GC $\times$ GC. With the developed method, hydrocarbons of up to nC60 as well as tetra-aromatic compounds in vacuum gas oil (VGO) could be eluted. Many groups have used HT-GC $\times$ GC to extend the carbon number range attainable by $\mathrm{GC} \times \mathrm{GC}$ and have optimised $\mathrm{HT}-\mathrm{GC} \times \mathrm{GC}$ parameters for the separation of aromatics, nitrogen-containing compounds as well as sulphurcontaining compounds in heavy petroleum fractions [12, 23 - 29].

In this study, the optimised HT-GC $\times$ GC column combinations for the separation of oxygenates in oxidised heavy paraffinic fractions are reported for the first time. The method is also applied to monitor the oxidation reaction of a hydrogenated heavy paraffinic fraction to illustrate the advantage of using $\mathrm{HT}-\mathrm{GC} \times \mathrm{GC}$, as compared to traditional titrimetric methods.

\section{EXPERIMENTAL}

\subsection{Chemicals}

Analytical gases for the HT-GC×GC were obtained from Afrox (South Africa). Cyclohexane was obtained from Sigma Aldrich (St Louis, MO, USA) and a hydrogenated heavy paraffinic fraction was obtained from Sasol Wax (Sasolburg, South Africa) for the oxidation experiments.

\subsection{Oxidation of a heavy paraffinic fraction}

The oxidised heavy paraffinic fraction sample was prepared according to the procedure described by Breet et al. [30]. A bubble column type reactor that consists of a Pyrex glass column was used to oxidise the heavy paraffinic fraction with air containing $21.2 \%$ oxygen. The air entered the reactor through a frit to ensure proper sparging and improved oxidation. The temperature within the reactor was regulated with a contact thermometer. The reactor was loaded with $800 \mathrm{~g}$ of the hydrogenated 
heavy paraffinic fraction sample and air was passed through the system at a low flow rate until the required oxidation temperature was reached $\left(160^{\circ} \mathrm{C}\right)$. The air flow rate was then adjusted with a rotameter to $1.8 \mathrm{~L} / \mathrm{min}$ for the remainder of the experiment. The reactor was kept at this temperature for $300 \mathrm{~min}$. The reaction product was used to evaluate different column combinations.

In addition, an oxidation reaction was monitored at different times during the reaction. The start of the experiment $\left(\mathrm{t}_{0}\right)$ was defined as the time when the desired temperature was reached and the air flow was increased. Samples were then taken with a glass tube from the reactor at 30,60, 120, 180 and $210 \mathrm{~min}$ intervals during a single oxidation reaction.

\section{3 $\mathrm{GC} \times \mathrm{GC}$ method}

A Pegasus 4D GC×GC from Leco Corporation (St. Joseph, USA) equipped with a cryogenic $\mathrm{N}_{2}$ dual jet modulator and time-of-flight mass spectrometer (TOFMS) was used for all column set evaluation experiments and TOFMS spectra were collected between 35 and $1000 \mathrm{~m} / \mathrm{z}$ at 100 spectra/s. A cool-on-column injector (Agilent Technologies, Little Falls, USA) was used on this system. The oxidised heavy paraffinic fraction samples $(20 \mathrm{mg})$ were diluted in cyclohexane $(10 \mathrm{ml})$ prior to injection and $0.5 \mu \mathrm{L}$ of this solution was injected for all experiments. Data analysis was performed using ChromaTOF software (Leco, v4.50.8.0). Different combinations of non-polar and medium polarity columns were evaluated and these are presented in Table 1. All the experiments were carried out using helium as a carrier gas at a constant flow rate close to the optimum velocity of the first dimension column [31]. The HT-GC $\times$ GC oven was operated at programmed temperatures starting in all cases at $100^{\circ} \mathrm{C}$ and increasing at $3^{\circ} \mathrm{C} / \mathrm{min}$ to the maximum temperature of the column set (Table 1) and kept constant at the final temperature for $5 \mathrm{~min}$. The liquid $2 \mathrm{~L} \mathrm{~N}_{2}$ dewar supplying the cryogenic modulator was filled only once at the start of the analysis. This resulted in the gradual evaporation of the liquid nitrogen from the dewar from start of the analysis until 60 min, after which the cooling jets continued at room temperature. The modulation period was set to $6 \mathrm{~s}$ in all cases. 
Table 1. Evaluated column sets for the HT-GC×GC experiments.

\begin{tabular}{|c|c|c|c|c|}
\hline Set & Configuration & First Column & Second Column & Max Temp \\
\hline 1 & Reversed & Trb50ht $(30 \mathrm{~m} \times 0.32 \mathrm{~mm}, 0.1 \mu \mathrm{m})$ & BPX-1 $(1.0 \mathrm{~m} \times 0.1 \mathrm{~mm}, 0.1 \mu \mathrm{m})$ & $370^{\circ} \mathrm{C}$ \\
\hline 2 & Reversed & Trb50ht $(30 \mathrm{~m} \times 0.25 \mathrm{~mm}, 0.1 \mu \mathrm{m})$ & BPX-1 $(1.0 \mathrm{~m} \times 0.1 \mathrm{~mm}, 0.1 \mu \mathrm{m})$ & $370^{\circ} \mathrm{C}$ \\
\hline 3 & Reversed & Trb50ht $(10 \mathrm{~m} \times 0.32 \mathrm{~mm}, 0.1 \mu \mathrm{m})$ & BPX-1 $(0.5 \mathrm{~m} \times 0.1 \mathrm{~mm}, 0.1 \mu \mathrm{m})$ & $370^{\circ} \mathrm{C}$ \\
\hline 4 & Reversed & Trb50ht $(10 \mathrm{~m} \times 0.25 \mathrm{~mm}, 0.1 \mu \mathrm{m})$ & BPX-1 $(0.5 \mathrm{~m} \times 0.1 \mathrm{~mm}, 0.1 \mu \mathrm{m})$ & $370^{\circ} \mathrm{C}$ \\
\hline 5 & Normal & DB1HT $(30 \mathrm{~m} \times 0.32 \mathrm{~mm}, 0.1 \mu \mathrm{m})$ & Trb50ht $(1.5 \mathrm{~m} \times 0.1 \mathrm{~mm}, 0.1 \mu \mathrm{m})$ & $370^{\circ} \mathrm{C}$ \\
\hline 6 & Normal & DB1HT $(10 \mathrm{~m} \times 0.32 \mathrm{~mm}, 0.1 \mu \mathrm{m})$ & Trb50ht $(0.5 \mathrm{~m} \times 0.1 \mathrm{~mm}, 0.1 \mu \mathrm{m})$ & $370^{\circ} \mathrm{C}$ \\
\hline 7 & Normal & DB1HT $(10 \mathrm{~m} \times 0.53 \mathrm{~mm}, 0.15 \mu \mathrm{m})$ & Trb50ht $(0.5 \mathrm{~m} \times 0.1 \mathrm{~mm}, 0.1 \mu \mathrm{m})$ & $370^{\circ} \mathrm{C}$ \\
\hline
\end{tabular}

Column set 2 was selected for the analysis of the samples taken at different times during the oxidation reaction. A constant flow rate of $1.4 \mathrm{~mL} / \mathrm{min}$ was used and the oven starting temperature was $100^{\circ} \mathrm{C}$ and increased at $3^{\circ} \mathrm{C} / \mathrm{min}$ to $370^{\circ} \mathrm{C}$ and kept constant at this maximum temperature for $5 \mathrm{~min}$. A flame ionization detector (FID) was used to perform quantitative HT-GC $\times$ GC analyses of these oxidised samples. HT-GC [32] confirmed that more than $99 \%$ of the oxidised heavy paraffinic fraction sample and $97 \%$ of the hydrogenated heavy paraffinic fraction sample eluted within the range attainable by $\mathrm{HT}-\mathrm{GC} \times \mathrm{GC}(<\mathrm{C} 60)$. The carbon number distributions as determined by HT-GC is presented in Figure 1. Relative quantification was performed by normalization of peak areas obtained from the FID detector and a response factor of unity was assigned to hydrocarbons. Relative response factors were used for oxygenated compounds as determined from the analysis of oxygenated standards.

\section{HT-GC analysis}

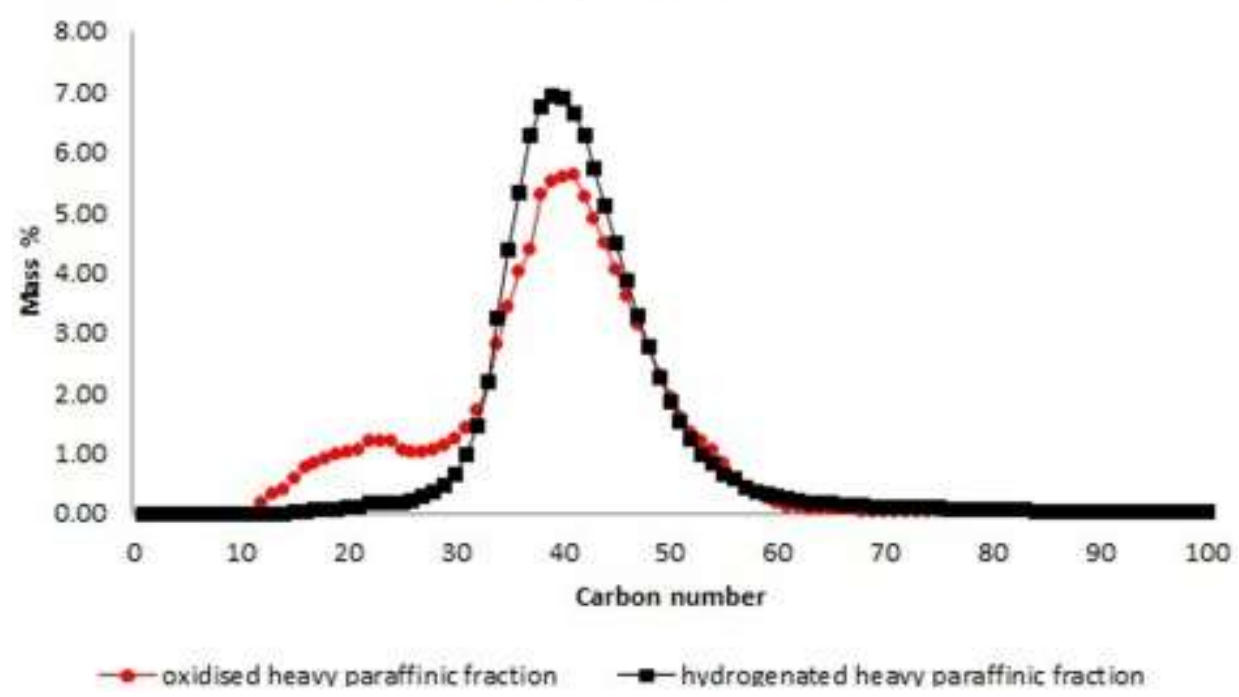

Fig. 1. Carbon number distributions for the oxidised heavy paraffinic fraction and the hydrogenated heavy paraffinic fraction as determined by HT-GC. 


\section{RESULTS AND DISCUSSION}

\subsection{Selection of 2D configurations}

Of all the chromatographic conditions described above, the selected combination of GC columns is probably the most critical for the analysis of heavy paraffinic fractions. $\mathrm{GC} \times \mathrm{GC}$ and $\mathrm{HT}-\mathrm{GC} \times \mathrm{GC}$ analysis of fuels and heavy petroleum fractions is commonly reported using a normal phase configuration [12, 26, 29, 33 -34], with a non-polar column in the $1^{\text {st }}$ dimension. Polydimethylsiloxane phases are commonly used in onedimensional high temperature applications [35] since they have very good thermal stability and easily elute hydrocarbons. Agilent DB1HT (Little Falls, USA) and SGE BPX-1 (Ringwood, Australia) columns were therefore selected to as the non-polar phases for the different column sets (Table 1).

Several groups $[33,36-39]$ showed that the highest orthogonality in $G C \times G C$ for products containing alkanes, alkenes, aromatics and oxygenates is obtained in the reversed column configuration with a polar column in the $1^{\text {st }}$ dimension. The columns used by these authors cannot be applied to the HT-GC×GC analysis of heavy oxidised paraffinic fractions due to the reduced upper temperature limits of polar columns. As reported in previous $H T-G C \times G C$ column set evaluations [12, $23-29$ ], a mid polarity column with a higher temperature stability was used in this study. Dutriez et al. [12] used a (50\% Phenyl)polysilphenylene-siloxane column from SGE (Victoria, Australia) with a $370^{\circ} \mathrm{C}$ maximum programmable temperature. However, after a performance review by SGE, the maximum programmable temperature of this column was lowered to $350^{\circ} \mathrm{C}$. The TRB-50 column with (50\%) Diphenyl-(50\%)dimethyl polysiloxane phase from Teknokroma (Barcelona, Spain) with a $370^{\circ} \mathrm{C}$ maximum programmable temperature was therefore selected as the mid polar stationary phase for the different column sets (Table 1). As in other studies [12, 26 - 27] the selection of the column sets focused on columns of different lengths with a reduced film thickness and also on different normal and reversed configuration column combinations.

Gaines et al. [22] investigated the temperature requirements for the trapping and releasing of compounds in a cryogenic gas loop-type modulator and showed that excessive cold temperatures cause peak distortions for heavy compounds (> C26). To avoid these excessive cold temperatures during the analysis of heavy hydrocarbons with a liquid $\mathrm{N}_{2}$ cryogenic modulator, the liquid $\mathrm{N}_{2}$ nitrogen dewar attached to the 
instrument was only filled at the start of the analysis. The gradual evaporation of the liquid $\mathrm{N}_{2}$ from the dewar resulted in the gradual increase in modulation temperature from start of the analysis until approx. 60 min of the analysis after which modulation continued at room temperature. No significant peak broadening along the first dimension was observed for heavy molecules as published by Gaines et al. [22] and the modulation produced very narrow peaks which indicated that the procedure of gradual evaporation was successful in limiting first dimension peak broadening due to cold modulator temperatures.

\subsection{Evaluation of 2D configurations}

The selectivity of the $2 \mathrm{D}$ column sets in Table 1 was compared to one another to determine which set provides the optimum separation of oxygenates in oxidised heavy paraffinic fractions. Similar to previous studies [26 - 27] where the optimum separation of sulphur- and nitrogen-containing compounds was investigated, the $2 \mathrm{D}$ selectivity was estimated by calculating the $2 \mathrm{D}$ resolutions $\left(\mathrm{Rs}_{2 \mathrm{D}}\right)$ for selected peaks. The $2 \mathrm{D}$ resolution method introduced by Giddings [20] was used where the 2D resolution, $\mathrm{Rs}_{2 \mathrm{D}}$, between solutes $\mathrm{A}$ and $\mathrm{B}$ is defined as the Euclidean norm of the resolution over the two axes (Eq. (1)),

$$
R s_{2 D}=\sqrt{{ }^{1} R s^{2}+{ }^{2} R s^{2}}
$$

where ${ }^{1} \mathrm{Rs}$ and ${ }^{2} \mathrm{Rs}$ are the respective resolutions between two compounds of interest along the first and the second dimensions. The $2 \mathrm{D}$ resolution can also be given as

$$
R s_{2 D}=\sqrt{\frac{4^{*}\left(\Delta^{1} \mathrm{t}_{R}\right)^{2}}{\left({ }^{1} \omega_{A}+{ }^{1} \omega_{B}\right)^{2}}+\frac{4^{*}\left(\Delta^{2} \mathrm{t}_{R}\right)^{2}}{\left({ }^{2} \omega_{A}+{ }^{2} \omega_{B}\right)^{2}}}
$$

where, ${ }^{1} \omega$ and ${ }^{2} \omega$ are the peak widths along each dimension at $10 \%$ peak height for compounds $A$ and $B$. The difference in retention times between the apices of the two 
compounds are depicted as $\Delta^{1} t_{R}$ and $\Delta^{2} t_{R}$. The first dimension peak widths $\left({ }^{1} \omega\right)$ are considered equal to the sum of the number of modulations for the compound of interest providing that the modulated peaks are higher than $10 \%$ of the largest modulation value. The second dimension peak widths $\left({ }^{2} \omega\right)$ at $10 \%$ peak height were measured on the chromatogram.

2D occupation was also used as criteria to determine which column set provides the optimum separation of oxygenates and was calculated to quantify the spreading on each 2D contour plot $[27,40]$. To calculate the 2D occupation, the first and last eluted peaks in each dimension was considered and the occupation was calculated according to Eq. (3).

$$
\text { 2D Occupation }=\frac{{ }^{1} \operatorname{tr}_{a}-{ }^{1} \operatorname{tr}_{b}}{{ }^{1} \mathrm{Tr}} \times \frac{{ }^{2} \operatorname{tr}_{c}-{ }^{2} \operatorname{tr}_{d}}{\mathrm{P}_{\text {mod }}}
$$

where, ${ }^{1} \operatorname{tr}_{a}$ is the first dimension retention time of the peak that eluted last in the first dimension, ${ }^{1} \operatorname{tr}_{\mathrm{b}}$ is the first dimension retention time of the peak that eluted first in the first dimension, ${ }^{2} \mathrm{tr}_{\mathrm{c}}$ is the second dimension retention time of peak that eluted last in the second dimension, ${ }^{2} \operatorname{tr}_{d}$ is the second dimension retention time of the peak that eluted first in the second dimension, ${ }^{1} \mathrm{Tr}$ is the total run time or total first dimension analysis time and $\mathrm{P}_{\text {mod }}$ is the $2 \mathrm{D}$ modulation period.

The sample prepared for the column set evaluation was analysed with the column sets presented in Table 1. The 2D contour plots for the reversed column configurations and the $2 \mathrm{D}$ contour plots for the normal column configurations were very similar and therefore only the $2 \mathrm{D}$ contour plots of the first column set of each configuration is presented. The 2D contour plot obtained with Column Set 1 is shown in Figure 2. 


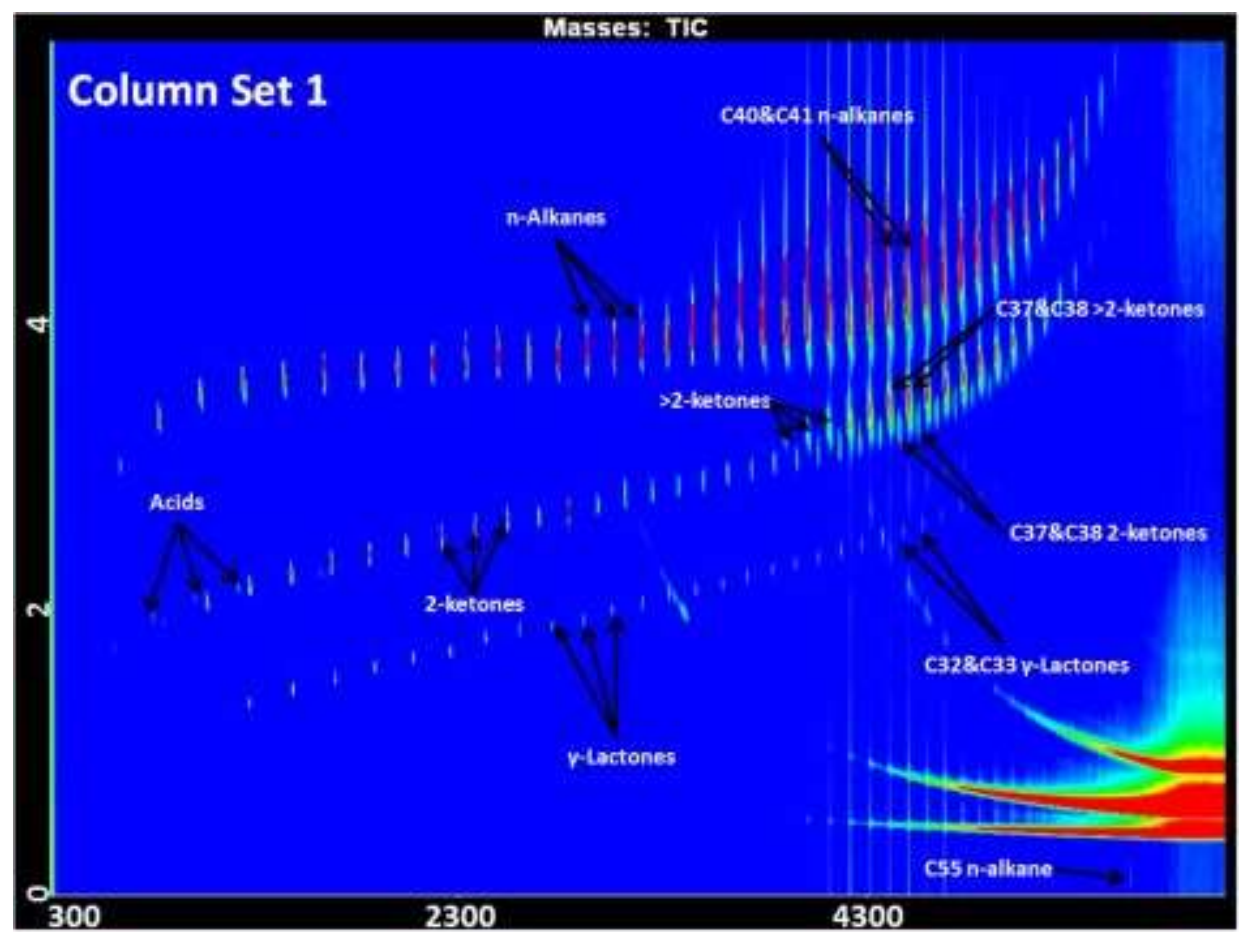

Fig. 2. $2 \mathrm{D}$ contour plot of the oxidised heavy paraffinic fraction corresponding to column Set 1 .

A highly ordered 2D chromatogram was obtained and the two-dimensional separation space was well utilized. The separation in the two-dimensional separation plane occurs according to boiling point as well as polarity differences and n-alkanes up to a carbon number of nC60 (b.p. $615^{\circ} \mathrm{C}$ ) are eluted in the first dimension with the paraffins from nC55 eluting in "wrap-around", i.e. with more than one period of modulation. Separation is also observed between n-alkanes and different classes of oxygenates in the second dimension. These prominent oxygenates were identified by mass spectra library [41] matching as carboxylic acids, 2-ketones and y-lactones. Ketones with the carbonyl functionality further along the hydrocarbon chain than position 2 were also identified and since these ketones could not be accurately identified from the mass spectra, they were named $>2$-ketones. It was mentioned that in $\mathrm{GC} \times \mathrm{GC}$ peaks are often arranged in highly ordered and structured plots, where peaks belonging to homologous series are positioned along straight lines on the retention plane and one can clearly distinguish the different classes of oxygenates eluting below the paraffins with the more polar oxygenate classes having the shortest second dimension retention times [33]. When using the reversed $\mathrm{GC} \times \mathrm{GC}$ configuration, the separation of oxygenates in the second dimension is very much a function of the polarity of the first dimension column since the more polar oxygenates have longer first dimension retention times compared to the non-polar alkanes. These oxygenates therefore elute 
from the first dimension column together with slightly higher boiling alkanes and due to differences in their volatilities, these compounds are then separated very well in the second dimension [42].

The identified oxygenates are consistent with the typical oxygenates that are formed during the autoxidation of saturated hydrocarbons with molecular oxygen which is a free radical chain process. Goosen et al. [7] have shown that the autoxidation of nonane and decane occurs in three stages with the initial stage leading to alkyl hydroperoxide formation. The alkyl hydroperoxide decomposes during the second stage to produce alcohols and ketones as major products. In the third stage the products are oxidised in competition with the alkanes. With the applied conditions of their autoxidation study, the alkanes produced $\mathrm{y}$-lactones as the major ester components and carboxylic acids were formed in the latter stages of oxidation. Other studies have also reported the formation of these species [7 - 9].

In this study the intra-family resolutions for all the reversed configuration column sets were obtained by calculating the 2D resolutions between C40 and C41 alkanes, C37 and C38 2-ketones, C37 and C38 >2-ketones and C32 and C33 y-lactones.

In order to calculate the inter-family resolutions the concept of neighbouring peaks needs to be established since it is very different for two-dimensional chromatography compared to one-dimensional chromatography. According to the work by Pieters et al. [43], one peak in a two dimensional chromatogram may be surrounded by more than two neighbouring peaks and a method was proposed to determine actual neighbouring peaks. The method involves the connection of lines between all the 1D peak maxima of two peaks of interest (peak $A$ and peak $B$ ). If all the connecting lines between peak $A$ and peak $B$ cross the peak region of a peak eluting between peak $A$ and peak $B$, they are not considered neighbours. In the case where not all or none of the connecting lines between the peak $A$ and peak $B$ cross the peak region of a peak eluting between peak $A$ and peak $B$, they are considered neighbours. Based on this description, the C40 alkane and C37 >2-ketone, C37 >2-ketone and C37 2-ketone, C40 alkane and C37 2-ketone and the C37 2-ketone and C32 y-lactone were selected to calculate the inter-family resolutions for all reversed configuration column sets (Table 1). These selected compounds were close to the middle of the carbon distribution of the oxidised heavy paraffinic fraction and therefore in the region of the 
chromatogram where peak overlap is most likely to occur and where optimum resolution between compounds is critical.

The 2D contour plot of the sample prepared for the column evaluations obtained with the normal column configuration set 5 (Table 1) is shown in Figure 3.

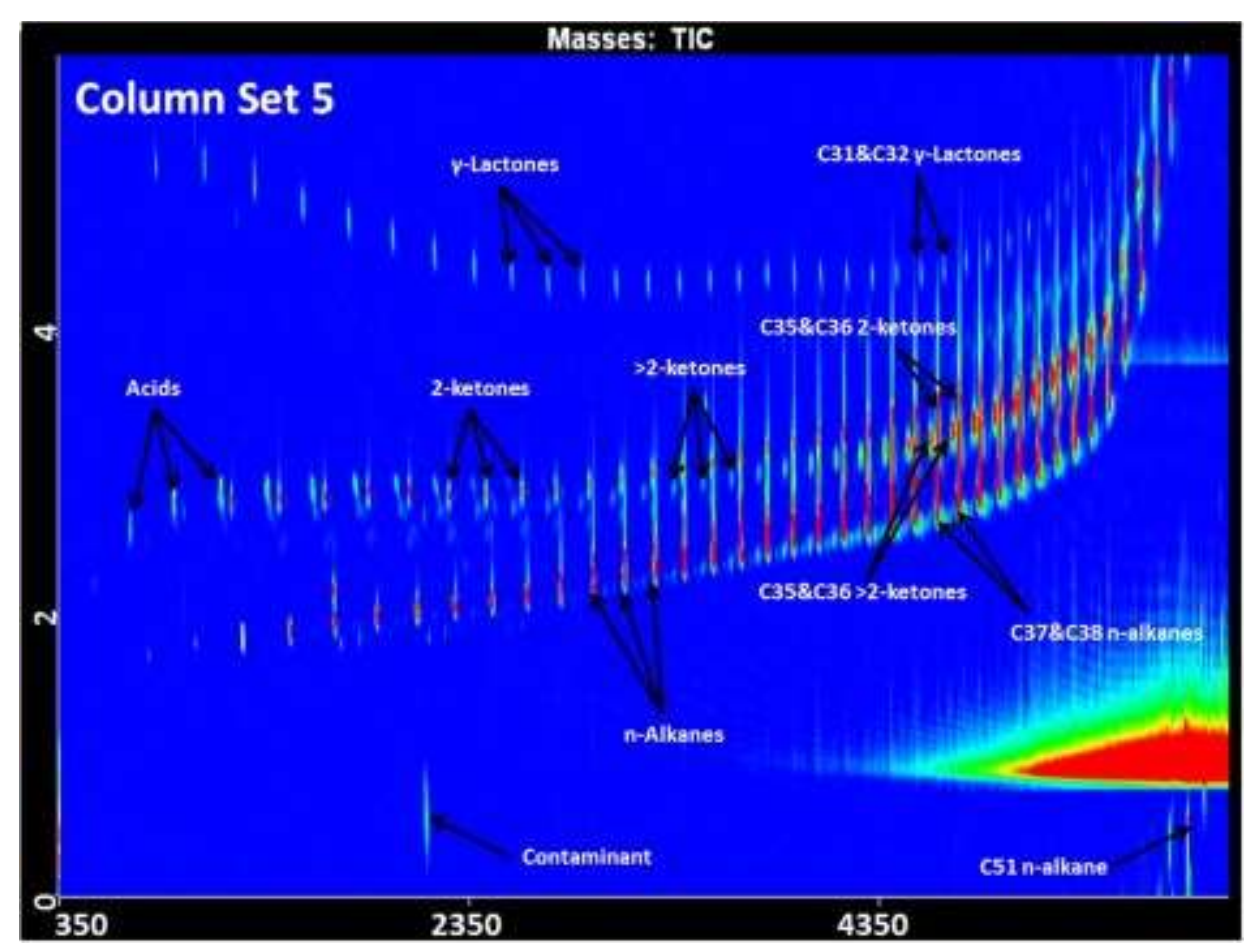

Fig. 3. $2 \mathrm{D}$ contour plot of the oxidised heavy paraffinic fraction corresponding to column Set 5.

The intra-family resolutions for all the normal configuration column sets were obtained by the calculation of the 2D resolutions between C37 and C38 alkanes, C35 and C36 2-ketones, C35 and C36 >2-ketones and C31 and C32 y-lactones. Based on the description of neighbouring peaks by Pieters et al. [43], the inter-family resolutions for all the normal configuration column sets (Table 1) were calculated between the C37 alkane and C35 >2-ketone, C35 >2-ketone and C35 2-ketone, C37 alkane and C35 2ketone and between the C35 2-ketone and C32 $\mathrm{y}$-lactone. Once again, these compounds were close to the middle of the carbon distribution of the oxidised heavy paraffinic fraction and the region of the chromatogram where peak overlap will most likely occur. 
The intra-resolutions for the column sets (Table 1) are presented in Figure 4.

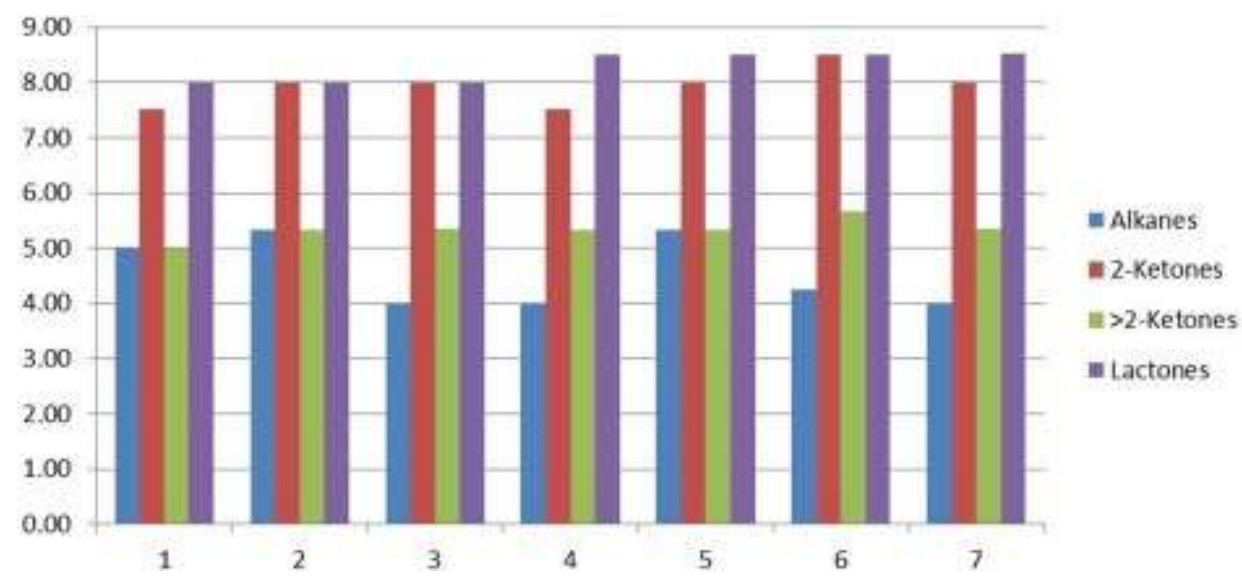

Fig. 4. Intra-family resolutions for the evaluated column sets.

Based on 1DGC convention, $\mathrm{Rs}_{2 \mathrm{D}}$ is sufficient when it is higher than 1.5 [27]. Figure 4 indicated that intra-family resolutions were very similar for all column sets and in all cases the resolutions were higher than 1.5 and therefore the intra-family resolutions were deemed sufficient for all the investigated column sets.

The inter-family resolutions for the column sets (Table 1) are presented in Figure 5.

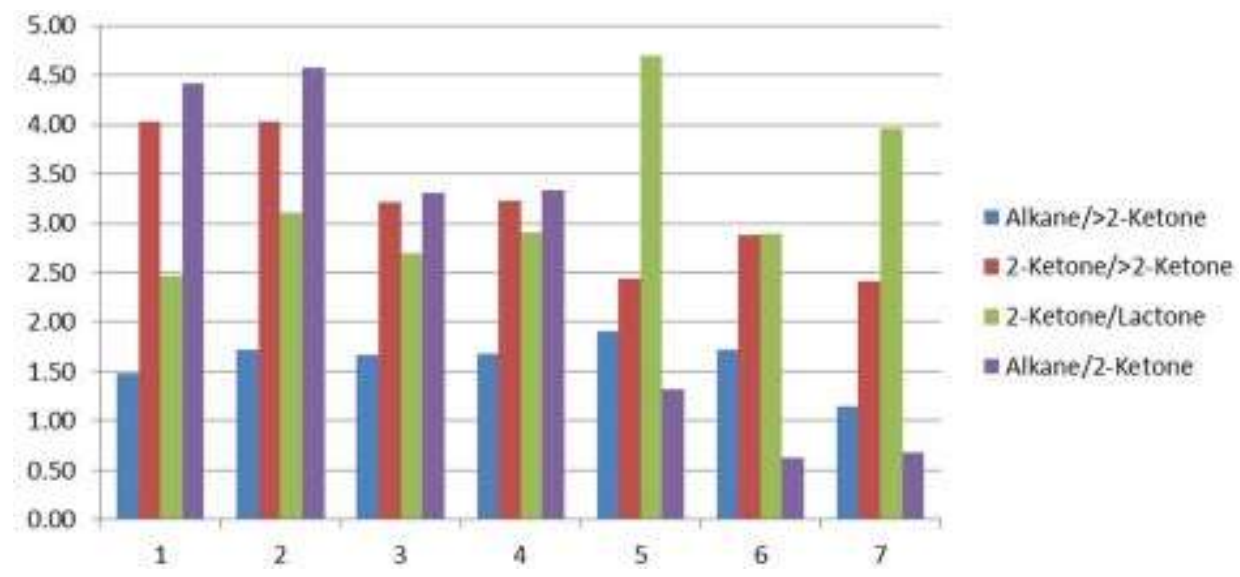

Fig. 5. Inter-family resolutions for the evaluated column sets.

Figure 5 indicates that inter-family resolutions between the alkane and the $>2$-ketone were close to the required 1.5 for all the evaluated column sets with column set 1 and set 7 having resolutions below the required 1.5. Slightly better resolutions between the alkane and the $>2$-ketones were obtained by column sets 2 - 6 . The resolutions between the 2-ketone and $>2$-ketone as well as the resolutions between the 2-ketone and $y$-lactone were sufficient for all the column sets. Insufficient resolutions were 
obtained between the alkane and 2-ketone for all the normal configuration column sets (Sets 5 - 7) whilst all the reversed phase configurations (Sets 1 - 4) showed sufficient resolutions with column set 2 having the highest resolution between these two compounds. The resolution between the alkane and 2-ketone was more than 2 times higher with the reversed column configuration column sets than that achieved with the normal column configuration column sets. In the case of the reversed column configuration, the $n$-alkanes eluted at the top of the separation plane and the large $2^{\text {nd }}$ dimension peak widths of the $n$-alkanes, due to their high concentrations, caused fractions of these $n$-alkane peaks to elute at the bottom of the separation plane (wraparound). No other oxygenates have $2^{\text {nd }}$ dimension retention times in the same region and therefore the overlap between the alkanes and 2-ketones in normal configuration were not observed in the reversed column configurations. The resolution between these compounds classes was therefore viewed as critical in this evaluation and weighed heavily in the choice of a column set that provides optimal separation of the compounds present.

The 2D occupations for the different column sets are presented in Figure 6.

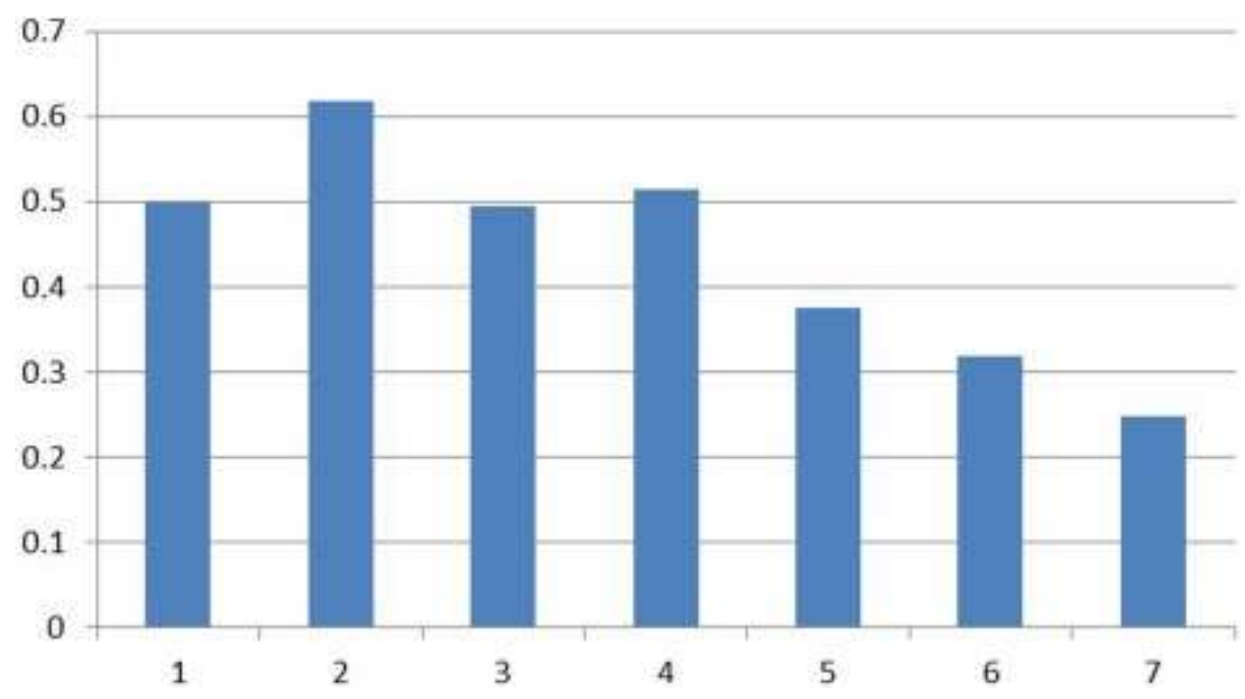

Fig. 6. Comparison of 2D occupation for the different column sets.

In Figure 6 it is clear that the reversed configuration column sets (sets $1-4$ ) provide the best $2 \mathrm{D}$ occupations with the normal configuration column sets providing lower $2 \mathrm{D}$ occupations in each case. Previous groups that focussed on the optimal separation of FT-products that contained mostly alkanes, alkenes, aromatics and oxygenates also 
showed that the highest orthogonality for these products is obtained in the reversed column configuration [33, 36 - 39]. Based on the above discussion, column set 2 was chosen as the optimum column set for the separation of oxygenates in oxidised heavy paraffinic fractions.

\subsection{Oxidation Reaction Monitoring}

As mentioned previously, the characterization and monitoring of heavy oxygenate formation is very important for the control of oxidation processes. In order to illustrate the use of $\mathrm{HT}-\mathrm{GC} \times \mathrm{GC}$ in oxidation reaction monitoring, the hydrogenated heavy paraffinic fraction sample prior to oxidation as well as samples taken from the oxidation reactor during oxidation were analysed using column set 2 (Table 1).

The 2D contour plots of the hydrogenated heavy paraffinic fraction, prior to oxidation, as well as the oxidised products after 30,60,120, 180 and 210 min of oxidation are presented in Figure 6.

The increase in oxygenates with the increasing time intervals is clearly observed in Figure 6. In addition, the major classes of oxygenates are distinguishable in the chromatograms. The major classes of oxygenates were quantified (GC×GCFID) and the weight \% of the compounds present in the samples are presented in Table 2.

Table 2. Quantification results (GCxGCFID) of the heavy paraffinic fraction sample as well as the oxidised products.

\begin{tabular}{|l|c|c|c|c|c|c|}
\hline Sample & $\begin{array}{c}\text { Paraffins } \\
(\text { mass\%) }\end{array}$ & $\begin{array}{c}\text { 2-ketones } \\
(\text { mass\%) }\end{array}$ & $\begin{array}{c}\text { >2-ketones } \\
(\text { mass\%) }\end{array}$ & $\begin{array}{c}\text { V-lactones } \\
(\text { mass\% })\end{array}$ & $\begin{array}{c}\text { Acids } \\
(\text { mass\% })\end{array}$ & $\begin{array}{c}\text { Other } \\
(\text { mass\% })\end{array}$ \\
\hline Heavy paraffinic fraction & 100.00 & 0.00 & 0.00 & 0.00 & 0.00 & 0.00 \\
\hline $30 \mathrm{~min}$ & 89.97 & 3.02 & 5.71 & 0.96 & 0.02 & 0.32 \\
\hline $60 \mathrm{~min}$ & 83.88 & 4.28 & 8.23 & 2.72 & 0.15 & 0.74 \\
\hline $120 \mathrm{~min}$ & 69.76 & 7.43 & 14.99 & 4.77 & 0.41 & 2.64 \\
\hline $180 \mathrm{~min}$ & 61.38 & 9.21 & 17.91 & 6.70 & 0.73 & 4.07 \\
\hline $210 \mathrm{~min}$ (Final Product) & 51.87 & 9.37 & 18.17 & 9.78 & 3.97 & 6.84 \\
\hline
\end{tabular}

It can be seen from Table 2 that there is a steady increase in the oxygenate concentration with the final product after 210 min of oxidation containing 48.13 mass $\%$ 
of oxygenates. The $>2$-ketones were observed to be the oxygenate class with the highest concentration. Cyclic esters ( $y$-Lactones) were also observed in high concentrations and carboxylic acids were only formed in the latter stages of the oxidation reaction. This is consistent with the observations by Goosen et al. [7] in their study of the autoxidation of nonane and decane. Other minor oxidation products were grouped together and the concentration of these oxygenates were 6.84 mass $\%$. The minor oxidation products were not identified as part of this study and more work is needed to identify these minor oxygenates. Contrary to previous studies [7 - 9], alcohols were only identified as a minor component in the oxidation products.

The advantage of using $\mathrm{HT}-\mathrm{GC} \times \mathrm{GC}$ over the use of titrimetric procedures in oxidation reaction monitoring is clearly illustrated in this example where the concentrations of different oxygenate classes could be determined selectively with the added advantage that the concentrations for each oxygenate class can be reported by carbon number. It is for example clear that the carbon number distribution of the alkanes changes with time and that each oxygenate class has a different carbon number distribution. Another advantage is that the separate titrimetric procedures can be replaced by a single HT$\mathrm{GC} \times \mathrm{GC}$ analysis to monitor oxidation reactions. The developed $H T-G C \times G C$ method can only be used for heavy paraffinic fractions with a carbon number distribution up to nC60 (b.p. $615^{\circ} \mathrm{C}$ ) which is similar to the carbon number distribution eluted in other studies [12] where column sets with similar geometries were used. Peak tailing in the second dimension is still observed for the alkanes present in high concentrations (Figure 7). The use of reverse a fill/flush (RFF) differential flow modulator [44] can be investigated in future to reduce the observed tailing which will also improve quantification. 


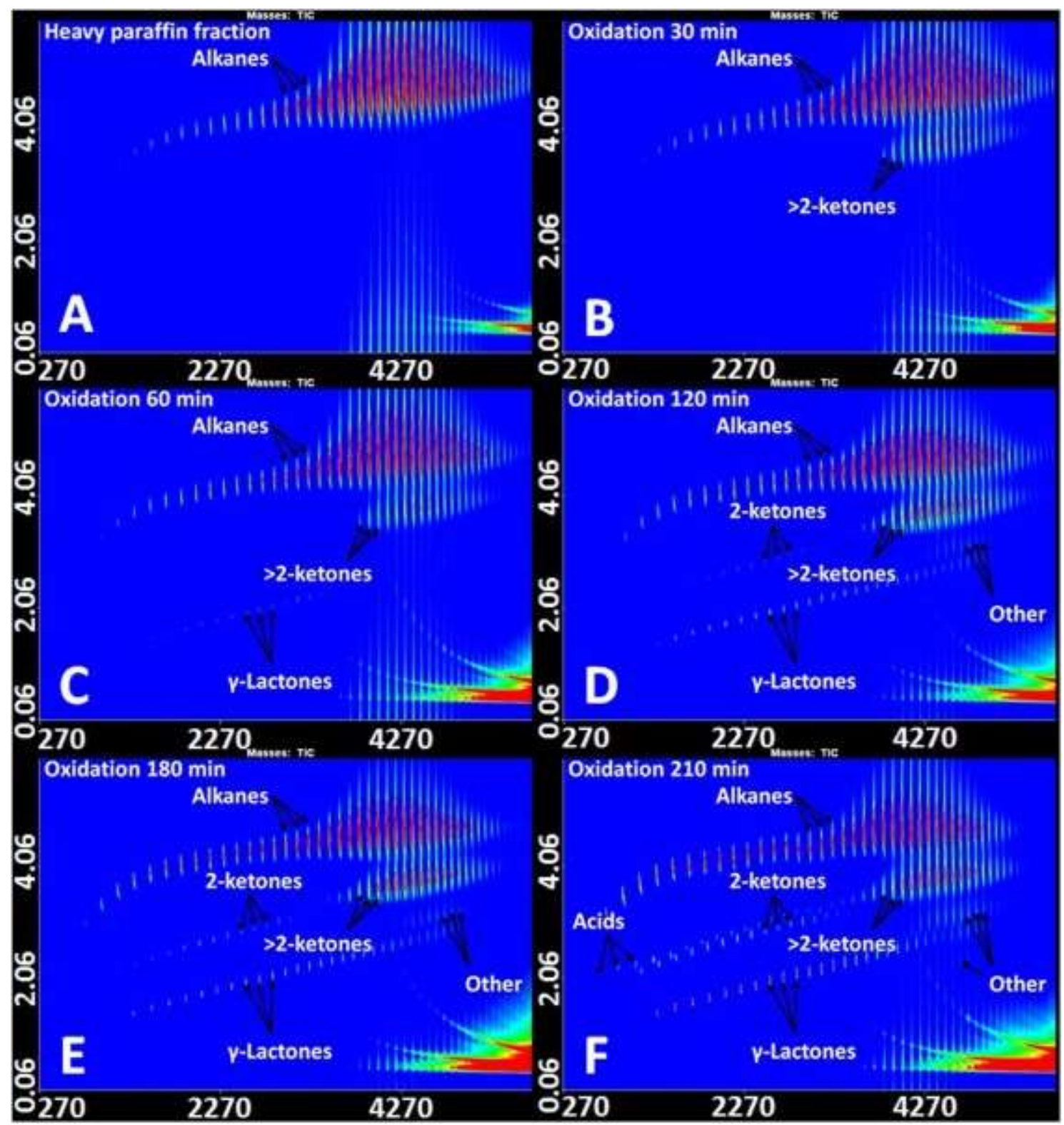

Fig. 7. $2 \mathrm{D}$ contour plots of the heavy petroleum fraction A) prior to oxidation B) After $30 \mathrm{~min}$ C) 60 min D) $120 \mathrm{~min}$, E) $180 \mathrm{~min}$ and F) 210 min of oxidation.

\section{CONCLUSIONS}

The evaluation of HT-GC×GC column sets for the optimum separation of oxygenates in oxidised heavy paraffinic fractions is reported for the first time. HT-GC $\times G C$ in the reversed configuration provides the optimum separation for oxygenates in oxidised heavy paraffinic fraction samples and hydrocarbons up to a carbon number of nC60 could be eluted. The advantages of using the developed HT-GC×GC method in the monitoring of the oxidation reactions of heavy paraffinic fraction samples were clearly illustrated and includes the selective quantification of oxygenate classes and obtaining the concentration for each oxygenate class by carbon number. Replacing separate 
titrimetric procedures by a single $H T-G C \times G C$ analysis also reduces the time and effort of analysis. HT-GCxGC analysis therefore allows for the optimization of oxidation conditions to produce higher value heavy paraffinic fractions. Future work may include the pre-separation and identification of the minor oxygenates classes. The use of reverse a fill/flush (RFF) differential flow modulator can also be investigated to reduce the observed tailing and to improve quantification. The repeatability of the quantitative analysis and the use of an internal standard quantification method will also be studied.

\section{REFERENCES}

[1] A. Steynberg, M.E. Dry, Introduction to Fischer-Tropsch Technology, Stud. Surf. Sci. Catal. 152 (2004) 406 - 481.

[2] A. de Klerk, Fischer-Tropsch Refining, Ph.D. Thesis, University of Pretoria, 2008.

[3] G.V. Webber, The Origin of Multiple DSC Melting Peaks of Fischer-Tropsch Hard Waxes, Ph.D. Thesis, University of Cape Town, 2009.

[4] D664. Standard Test Method for Acid Number of Petroleum Products by Potentiometric Titration, ASTM International. West Conshohocken. USA.

[5] D94. Standard Test Methods for Saponification Number of Petroleum Products, ASTM International. West Conshohocken. USA.

[6] E1899. Standard Test Method for Hydroxyl Groups Using Reaction with p-Toluenesulfonyl Isocyanate (TSI) and Potentiometric Titration with Tetrabutylammonium Hydroxide, ASTM International. West Conshohocken. USA.

[7] A. Goosen, D. H. Morgan, Autoxidation of Nonane and Decane: A Product Study, J. Chem. Soc. Perkin Trans. 2 (1994) 557 - 562.

[8] E.L.J. Breet, A.S. Luyt, Oxidation kinetics of a Fischer-Tropsch wax. Part 2. Induction period as first macroscopic process stage, S. Afr. J. Chem. 44 (4) (1991) 101 - 104.

[9] A. de Klerk, Continuous-Mode Thermal Oxidation of Fischer-Tropsch Waxes, Ind. Eng. Chem. Res. 42 (2003) 6545 - 6548.

[10] A. Sironi, G.R. Verga, Chapter 6, The O-FID and its applications in petroleum product analysis, in: E.R. Adlard (Ed.) Journal of Chromatography Library Series, Vol. 56, Elsevier, 1995, pp. $143-158$.

[11] C.C. Geng, S.Y. Li, Y. Ma, C.T. Yue, J.L. He, W.Z. Shang, Analysis and identification of oxygen compounds in Longkou shale oil and Shenmu coal tar, Oil Shale, 29 (2012) 322 - 333.

[12] T. Dutriez, M. Courtiadea, D. Thiébaut, H. Dulot, F. Bertoncinia, J. Vial, M. Hennion, Hightemperature two-dimensional gas chromatography of hydrocarbons up to nC60 for analysis of vacuum gas oils, J. Chromatogr A 1216 (2009) 2905 - 2912. 
[13] E. Kaal, H.G. Janssen, Extending the molecular application range of gas chromatography, J. Chromatogr. A 1184 (2008) 43 - 60.

[14] R.J.Wells, Recent advances in non-silylation derivatization techniques for gas chromatography J. Chromatogr. A 843 (1999) 1 - 18.

[15] K.L. Sobeih, M. Baron, J. Gonzalez-Rodriguez, Recent trends and developments in pyrolysis-gas chromatography, J. Chromatogr. A 1186 (2008) 51 - 66.

[16] M.P.D. Lucia, High temperature gas chromatography on narrow bore capillary columns, J. High Resolut. Chromatogr. 15 (1992) 256 - 259.

[17] M.H.P.M. van Lieshout, H. Janssen, C.A. Cramers, M.J.J. Hetem, H.J.P. Schalk, Characterization of polymers by multi-step thermal desorption/programmed pyrolysis gas chromatography using a high temperature PTV injector, J. High Resolut. Chromatogr. 19 (1996) 193 - 199.

[18] K. Grob, G. Grob, Capiliary columns with immobilized stationary phases. Part 5: Determination of column bleeding; re-silylation, J. High Resolut. Chromatogr. 5 (1982) 349 354.

[19] A.B. Fialkov, A. Gordin, A. Amirav, Extending the range of compounds amenable for gas chromatography-mass spectrometric analysis, J. Chromatogr. A 991 (2003) 217 - 240.

[20] J.C. Giddings, Concepts and comparisons in multidimensional separation, J. High Resolut. Chromatogr. 10 (1987) 319 - 323.

[21] W. Rathbun, Programmed automation of modulator cold Jet flow for comprehensive twodimensional gas chromatographic analysis of vacuum gas oils, J. Chromatogr. Sci. 45 (2007) $636-642$.

[22] R.B. Gaines, G.S. Frysinger, Temperature requirements for thermal modulation in comprehensive two-dimensional gas chromatography, J. Sep. Sci. 27 (2004) 380 - 388.

[23] F. Adam, F. Bertoncini, N. Brodusch, E. Durand, D. Thiébaut, D. Espinat, M. Hennion. New benchmark for basic and neutral nitrogen compounds speciation in middle distillates using comprehensive two-dimensional gas chromatography, J. Chromatogr A 1148 (2007) 55 - 64.

[24] T. Dutriez, M. Courtiade, D. Thiébaut, H. Dulot, M. Hennion, Improved hydrocarbons analysis of heavy petroleum fractions by high temperature comprehensive two-dimensional gas chromatography, Fuel 89 (2010) 2338 - 2345.

[25] T. Dutriez, M. Courtiade, D. Thiébaut, H. Dulot, F. Bertoncini, M. Hennion. Extended characterization of a vacuum gas oil by offline LC-high-temperature comprehensive twodimensional gas chromatography, J. Sep. Sci. 33 (2010) 1787 - 1796.

[26] T. Dutriez, J. Borras, M. Courtiade, D. Thiébaut, H. Dulot, F. Bertoncini, M. Hennion. Challenge in the speciation of nitrogen-containing compounds in heavy petroleum fractions by high temperature comprehensive two-dimensional gas chromatography, J. Chromatogr A 1218 (2011) 3190 - 3199. 
[27] L. Mahé, T. Dutriez, M. Courtiade, D Thiébaut, H. Dulot, F. Bertoncini. Global approach for the selection of high temperature comprehensive two-dimensional gas chromatography experimental conditions and quantitative analysis in regards to sulfur-containing compounds in heavy petroleum cuts, J. Chromatogr A 1218 (2011) 534 - 544.

[28] B.M.F. Ávila, R. Pereira, A.O. Gomes, D.A. Azevedo. Chemical characterization of aromatic compounds in extra heavy gas oil by comprehensive two-dimensional gas chromatography coupled to time-of-flight mass spectrometry, J. Chromatogr A 1218 (2011) 3208 - 3216.

[29] L. Mahé, M. Courtiade, C. Dartiguelongue, J. Ponthus, V. Souchon, D. Thiébaut, Overcoming the high-temperature two-dimensional gas chromatography limits to elute heavy compounds, J. Chromatogr A 1229 (2012) 298 - 301.

[30] E.L.J. Breet, A.S. Luyt, S. Oranje, Oxidation kinetics of a Fischer-Tropsch wax. Part I. A simple method for measuring oxygen consumption, S. Afr. J. Chem. 43 (3/4) (1990) 83 - 86.

[31] J. Beens, U. A.Th. Brinkman, Comprehensive two-dimensional gas chromatography - a powerful and versatile technique, Analyst 130 (2005) 123 - 127.

[32] EWF METHOD 001/03. Standard Test Method for Analysis of Hydrocarbon Waxes by Gas Chromatography, European Wax Federation. Bruxelles. Belgium.

[33] R. Van der Westhuizen, R. Crous, A de Villiers, P. Sandra, Comprehensive twodimensional gas chromatography for the analysis of Fischer-Tropsch oil products, J. Chromatogr A 1217 (2010) 8334 - 8339.

[34] C. Vendeuvre, F. Bertoncini, L. Duval, J.Duplan, D. Thiébaut, M.-C. Hennion, Comparison of conventional gas chromatography and comprehensive two-dimensional gas chromatography for the detailed analysis of petrochemical samples, J. Chromatogr A 1056 (2004) 155 - 162.

[35] D 6352. Standard Test Method for Boiling Range Distribution of Petroleum Distillates in Boiling Range from $174^{\circ} \mathrm{C}$ to $700^{\circ} \mathrm{C}$ by Gas Chromatography, ASTM International. West Conshohocken. USA.

[36] Vendeuvre, R. Ruiz-Guerrero, F. Bertoncini, L. Duval, D. Thiebaut, M.-C. Hennion, Characterisation of middle-distillates by comprehensive two-dimensional gas chromatography $(\mathrm{GC} \times \mathrm{GC})$ : A powerful alternative for performing various standard analysis of middle-distillates, J. Chromatogr. A 1086 (2005) 21 - 28.

[37] B. Omais, N. Charon, M. Courtiade, J. Ponthus, D. Thiebaut, A novel analytical approach for oxygen speciation in coal-derived liquids, Fuel, 104 (2013) 805 - 812.

[38] B. Omais, M. Courtiade, N. Charon, D. Thiebaut, A. Quignard, M.C. Hennion, Investigating comprehensive two-dimensional gas chromatography conditions to optimize the separation of oxygenated compounds in a direct coal liquefaction middle distillate, Journal of Chromatography A, 1218 (2011) 3233 - 3240. 
[39] B. Omais, M. Courtiade, N. Charon, C. Roullet, J. Ponthus, D. Thiebaut, Using gas chromatography to characterize a direct coal liquefaction naphtha, Journal of Chromatography A, 1226 (2012) 61 - 70.

[40] C. Cordero, P. Rubiolo, B. Sgorbini, M. Galli, C. Bicchi, Comprehensive two-dimensional gas chromatography in the analysis of volatile samples of natural origin: A multidisciplinary approach to evaluate the influence of second dimension column coated with mixed stationary phases on system orthogonality, Journal of Chromatography A, 1132 (2006) 268 - 279.

[41] National Institute of Standards and Technology (NIST), "Mass Spectrometry Library version 2.0f, build Oct 22", U.S. Secretary of Commerce, 2009.

[42] B. Omais, M. Courtiade, N. Charon, S; Esnault, D. Thiebaut, Reversal of elution order in a single second dimension by changing the first column nature in comprehensive twodimensional gas chromatography, Journal of Chromatography A, 1255 (2012) 196 - 201.

[43] S. Peters, G. Vivó-Truyols, P.J. Marriott, P.J. Schoenmakers, Development of a resolution metric for comprehensive two-dimensional chromatography, J. Chromatogr. A, 1146 (2007) 232 - 241.

[44] C. Duhamel, P. Cardinael, V. Peulon-Agasse, R. Firor, L. Pascaud, G. Semard-Jousset, P. Giusti, V. Livadaris, Comparison of cryogenic and differential flow (forward and reverse fill/flush) modulators and applications to the analysis of heavy petroleum cuts by hightemperature comprehensive gas chromatography, J. Chromatogr. A, 1387 (2015) 95 - 103. 